\title{
Congestion Reduction in Wireless Mesh Networks
}

\author{
Payal Grover \\ M.Tech. Research Scholar \\ GZS PTU Campus, \\ Bathinda, Punjab
}

\author{
Paramjeet Singh, PhD \\ Associate Professor \\ GZS PTU Campus, \\ Bathinda, Punjab
}

\author{
Shaveta Rani, PhD \\ Associate Professor \\ GZS PTU Campus, \\ Bathinda, Punjab
}

\begin{abstract}
Wireless Mesh Networks are looming as a key technology for next generation of existing wireless networks. The network falls into the category of infrastructure less wireless networks that rely on high mobility and dynamic topology. Though mesh topology provides good reliability, market coverage and scalability, there are number of issues and challenges in WMN. Amongst all, the most prevailing issue is the problem of packet loss due to congestion. The routing protocol always uses the best shortest path for the transmission of data. Congestion occurs when each time the protocol chooses the same path. Congestion problem can be overcome by involving a congestion control algorithm. In this paper, a congestion control scheme based on AODV routing protocol has been proposed. In this technique, buffers at the intermediate nodes are checked against two cases. First the capacity of the buffer on the whole is checked, if it crosses a certain threshold then second condition i.e. the capacity of the packets present in the buffer for the available destinations is checked against a second threshold. If it crosses a certain threshold value then data forwarding is carried out through alternate paths. The proposed technique has improved the performance of AODV protocol. Simulation results for AODV carried out in NS-2 clearly show the increase in throughput and decrease in end to end delay.
\end{abstract}

\section{General Terms}

Routing Protocols, Congestion Control.

\section{Keywords}

Congestion, Routing, Wireless Mesh Network, AODV.

\section{INTRODUCTION}

Wireless Mesh Networks has emerged as a growing area of research for next-generation wireless networking. Wireless Mesh Networks is a communication network consists of radio nodes organized in a Mesh Topology. WMNs provide greater flexibility, reliability, and performance over conventional MANET. WMNs can be integrated with other networks such as the Internet, cellular, IEEE 802.11, IEEE 802.15, IEEE 802.16 etc. through gateway and bridging functions in the mesh routers[1]. Wireless Mesh Networks follow a similar principle of operation, but unlike MANETs, the core of the network is assumed to be static. End-users are free to move and change their point of attachment to the mesh network at any time. WMNs consist of two types of nodes that are Mesh Routers and Mesh clients. Each node not only operates as a host but also as a router which forwards data on behalf of other nodes which may not be within the direct wireless transmission range of their destination nodes. As WMNs are self-organized networks, an ad-hoc network is automatically established by the nodes and maintains mesh connectivity. The gateway/bridge functionalities in mesh routers enable the integration of WMNs with various existing wireless networks such as cellular, Wi-Fi, wireless sensor networks.

Wireless mesh networks communicate by sending data packets over multiple wireless hops. These networks provide capabilities that facilitate the deployment of multi-hop wireless networks with access to the Internet. Mesh nodes can have multiple wireless interfaces in order to increase the capacity of the mesh network[2]. Mesh routers generally have minimal mobility and their main purpose is the formation of mesh backbone for the mesh clients. Interference and channel contention has made routing In WMN a very challenging task. Limited bandwidth and other resources further impose restrictions on the amount of data to be sent. In WMN, congestion can occur at any intermediate node deteriorating the performance of the network. It is a situation when a link or a node is carrying excessive load that it aids to deteriorates the quality of service.

Congestion occurs mainly when the load on the network is greater than the capacity of the network. However, factors like change in topology, number of flows, traffic characteristics of flows and their routes as well as channel capacity and available transmission rate at physical layer[3]. In WMN, information is forwarded using the best possible routes to any destination and the best path can be determined using routing protocol. The congestion problem occurs when every time routing protocol determines the same best path due to which the traffic load occurs on the same path while the other path seldom used, leading to packet loss hence degrading the performance of the network. This paper focuses on controlling congestion specifically in AODV protocol.

\section{AD HOC ON DEMAND DISTANCE VECTOR ROUTING PROTOCOL}

AODV was designed for networks with ten to thousands of mobile nodes. AODV creates routes on demand i.e. when required by the source and maintains as long as the communication is ongoing. AODV performs unicasting as well as multicasting. AODV helps in preserving active routes and reduces stale routes as routes are maintained as long as necessary. AODV uses sequence numbers to keep the freshness of routes and they also provide loop freedom. AODV is a reactive routing protocol and works in three phases; route discovery, forward path setup and route maintenance. Route discovery process is initiated with a route request packet broadcasted over the network when a node wishes to send data to some destination. This packet contains Source IP address, source node current sequence number, destination IP address, destination sequence number[4]. The sequence number helps to avoid the possibility of forwarding the same packet more than once. The RREQ packet also contains broadcast ID number. This is incremented each time a source node uses RREQ and Broadcast id and source IP address forms a unique identifier for RREQ. Broadcasting is 
done via flooding. Once an intermediate node receives a RREQ, the node sets up a reverse route entry for the source node in its routing table. Reverse Route entry consists of Source IP address, source sequence number, no. of hops to source, IP address of node from which RREQ has received. Using the reverse route, a node can send an RREP to the source in two cases, either it itself is a destination or it is having a route to destination. In the latter case the sequence number of the node must be greater than the sequence number of the route request packet. RREP packet is unicasted to the source in both cases otherwise a new RREQ is broadcasted by the source. The nodes keep track of the source IP address and Broadcast Id of every packet and if an RREQ is received which is already processed by a node it is discarded.

In forward path discovery phase, forward pointers are set to the path through which RREP travels. On receiving RREP, the source node starts sending data packets. Source keeps on updating its routing information and uses the best route every time. Routes are maintained as long as data is transmitted through them, when data transmission stops routes are timed out and are deleted eventually[5]. When a link break occurs, RRER messages are generated under the route maintenance phase by the node which is exactly before the node where link break occurs informing the source node that now destination is not reachable. On receiving RRER, if source still wants a route to destination it reinitiates the route discovery process.

\section{RELATED WORK}

A lot of work has been done in this area and several researchers have contributed in the field of congestion and provide solutions to counterpart the problem.

In [6], a congestion adaptive routing protocol. The protocol works in six phases and helps in congestion reduction is proposed. In CRP, every node on the route when prone to be congested warns its previous node. The previous node uses a "bypass" route to bypass the congestion area to the first noncongested node on the primary route then the traffic is split on these two routes according to the probabilities assigned minimizing multiple paths reducing overhead.

In [7], a Mobile Agent Based congestion control scheme which includes routing algorithm and a flow control at network layer is proposed. In this scheme, neighbors of source nodes are made Mobile Agents which collects and distributes the information about the network congestion. The mobile agents traverse the available routes and calculate the total congestion metric which can be estimated from the obtained queue length and channel contention already calculated.

In [8], efficient congestion avoidance and routing algorithm framework in wireless mesh networks which can be achieved with Congestion Aware Ant Colony Optimization algorithm (CACO) is proposed. CACO study the use of biologically inspired agents to route the packets in WMNs. CACO uses Ant Mesh, a distributed interference-aware data forwarding algorithm which enables smart ants to concurrently perform the routing and data forwarding. This evaluates the load on the network in order to properly qualify the outgoing links.

In [5], a new protocol is introduced named Congestion Adaptive AODV routing Protocol (CA-AODV) to cope with the congestion situation effectively and increase the network performance also in case of highly intensive multimedia data. The protocol ensures the availability of primary routes as well as alternative routes. If congestion happens at the primary route, affected node notifies its previous node about the congestion then the data will be transmitted from alternate route to the destination by that node. The protocol works in three divisions; congestion status setup, route discovery process and route maintenance process.

In [9], an algorithm for congestion control and efficient bandwidth minimization in wireless mesh networks is proposed. In wireless mesh networks the efficiency of the bandwidth management depends largely on the congestion control mechanisms hence the algorithm Cross Layer Congestion Control (CLCC) has been developed on the basis of concept of cross layer optimization. This provides an efficient bandwidth management scheme for high speed video applications through congestion control mechanism. The algorithm provides the optimal path for data transmission by estimating the bandwidth of all the available paths. It works as first determining all possible paths between source and destination then determine the available bandwidth from a particular source to a destination.

In [10], an efficient Bandwidth Congestion Control Mechanism for wireless mesh networks is introduced. The available bandwidth is calculated using the efficient measurement technique called probing. Probing is the continuous transmission of control packets all over the network which monitors the state of the network and returns the information like traffic, probe return time, throughput and train length. Available bandwidth is calculated through all these obtained factors. Hence, the transmission of data packets will be done, depending on the bandwidth available. Now it is essential to calculate the congestion over the network, if it is there ECN will notify about it if it is greater than threshold value then congestion control scheme will be called otherwise queue the packets to successfully transmit the packets to destination.

In [11], an Efficient Congestion Adaptive Routing Protocol for mobile ad-hoc networks is proposed. The currently designed routing protocols are not congestion adaptive, when network becomes prone to congestion routing protocols take a significant overhead, routing delay and packet losses. ECARP is designed to cope up with the congestion adaptively. It ensures the high availability of alternative routes and reduces the rate of stale routes. ECARP is developed by increasing some parameters of the AODV routing protocol. The parameters such as active_route_time-out, route_reply_wait_time, reverse_route_life, TTL_start, TTL increment, TTL_threshold and delete_period.

\section{PROBLEM STATEMENT}

In this section, the problems that occur in Wireless Mesh Networks because of Congestion are pointed out. Congestion control and security are major issues in WMN. Congestion is the instability of the network which results in packet loss contributing to retransmission of packets which leads to bottleneck stage. It can also lead to packet drops, high delays and low throughput. Moreover, it is expensive to control congestion in terms of overhead and time.

\section{PROPOSED SOLUTION}

To overcome Congestion problem in Wireless Mesh Networks, an algorithm based on AODV routing protocol using buffers is designed. In this approach, AODV routing protocol is used to implement routing in the network. Congestion Control Algorithm is responsible for preventing the occurrence congestion as well as alleviating the impact on congestion on network throughout if occurs. Although these goals can be achieved by imposing limitation on the rate of access on the source nodes to the network resources, the 
congestion control scheme should ensure that a fair distribution of resources among nodes is maintained.

In the proposed algorithm, multiple source destination pairs sending and receiving data at the same time are taken. In this, the buffer availability of nodes is checked to detect congestion in the path. If the buffer value is less than a threshold, packets through that route are sent continuously but if it exceeds a certain threshold then second condition is checked i.e. checking the number of packets present in the buffer for each destination respectively, if it increases a certain threshold then an alternate path for that particular source destination pair is chosen otherwise the packets will be forwarded through that route.

In this way, proposed technique resolves the congestion problem and reduces the delay and increases the throughput thereby increasing the network performance on the whole.

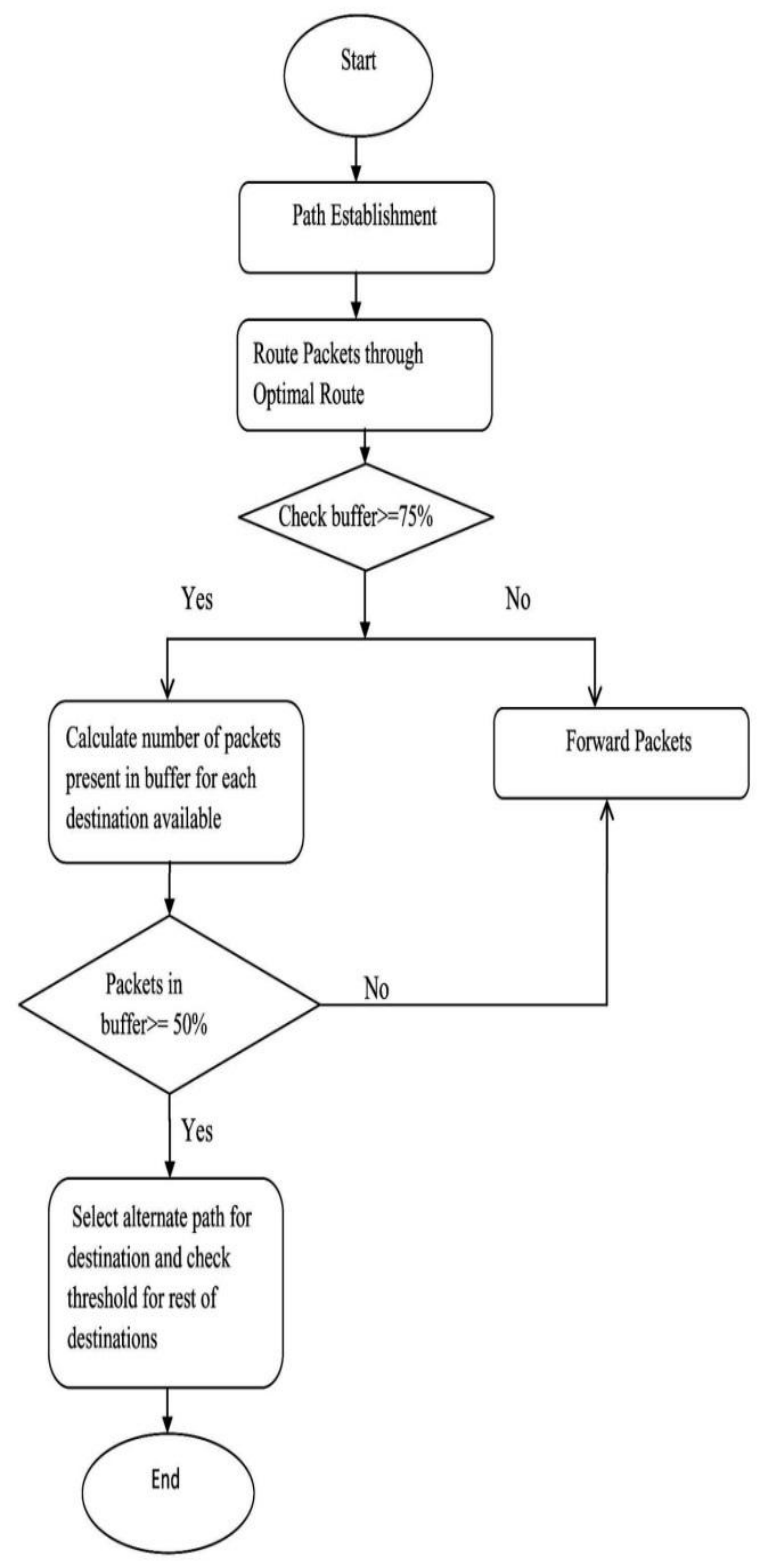

Figure 1: Flow Chart

\subsection{Proposed Algorithm}

Step 1: Consider multiple source destination pairs sending and receiving data at the same time.

Step 2: The route is established by broadcasting RREQ messages to all the neighboring nodes and the node having path to destination responds to RREQ by sending RREP back using unicasting to source node via intermediate nodes creating a path from source to destination.

Step 3: Route Packets and Check buffer availability at each intermediate node whether it is greater than or equal to a threshold value.

Step 4: If buffer space crosses a certain threshold value we will check for the second condition i.e. check number of packets in the buffer for particular destination.

Step 5: If the number of packets in the buffer for a particular destination is equal to or more than a certain threshold, consider an alternate path for transmission.

Step 6: Else forward packets through the same route.

Step 7: Repeat step 3 to 6 until all the destinations for which packets are available are covered.

The Diagrammatic Workflow of algorithm is given in Figure 1 .

\section{SIMULATION ENVIRONMENT}

The proposed protocol has been implemented in the simulated environment with NS-2. Network simulators provides a range of networking scenarios and in built protocols which makes it easier to implement various environments and get results. The routing protocol considered in this paper is AODV routing protocol. The simulation is carried out in a grid of area $1652 \mathrm{mx} 1652 \mathrm{~m}$. The experiments are performed in different by varying the number of nodes to get an idea of the network state for the performance metrics. Table 1 shows the simulation parameters which makes the scenario for the routing protocol.

Table 1. Simulation Parameters used for Simulation

\begin{tabular}{|c|c|}
\hline Simulator used & NS-2 \\
\hline Number of Nodes & $5,10,15,20,40$ \\
\hline Simulated Area & $1652 \mathrm{mx} 1652 \mathrm{~m}$ \\
\hline Simulation Time & $111 \mathrm{sec}$ \\
\hline Routing Protocol & AODV \\
\hline Traffic Type & CBR/FTP \\
\hline $\begin{array}{c}\text { Packet Size } \\
\text { Node Movement at } \\
\text { maximum Size }\end{array}$ & 1024 bytes \\
\hline
\end{tabular}




\subsection{Performance Metrics}

1. Throughput: It is the rate of delivery of packets i.e. the number of packets delivers in time. It is measured in kbps. Its value must be increased so as to increase the performance of the network.

2. End to End Delay: It is the average time taken by a packet to reach from source to destination. It is calculated by subtracting "time at which first packet was transmitted by source from time at which first data packet arrived to destination. It is measured in seconds. It is expected to be minimized.

\section{SIMULATION RESULTS}

This section represents the simulation results that have been evaluated by implementing various simulation parameters considered earlier. The simulation is run against different environments by varying number of nodes against two different metrics to get an idea of the network and to measure performance of the network.

\subsection{Graphical Representation}

1. Throughput: The graph represents the throughput analysis with respect to number of nodes in AODV routing protocol. It is clear from the graph that the throughput of the Modified AODV is increasing as compared to the earlier AODV protocol. The proposed system gives better results in case of throughput hence increases the performance of the network.

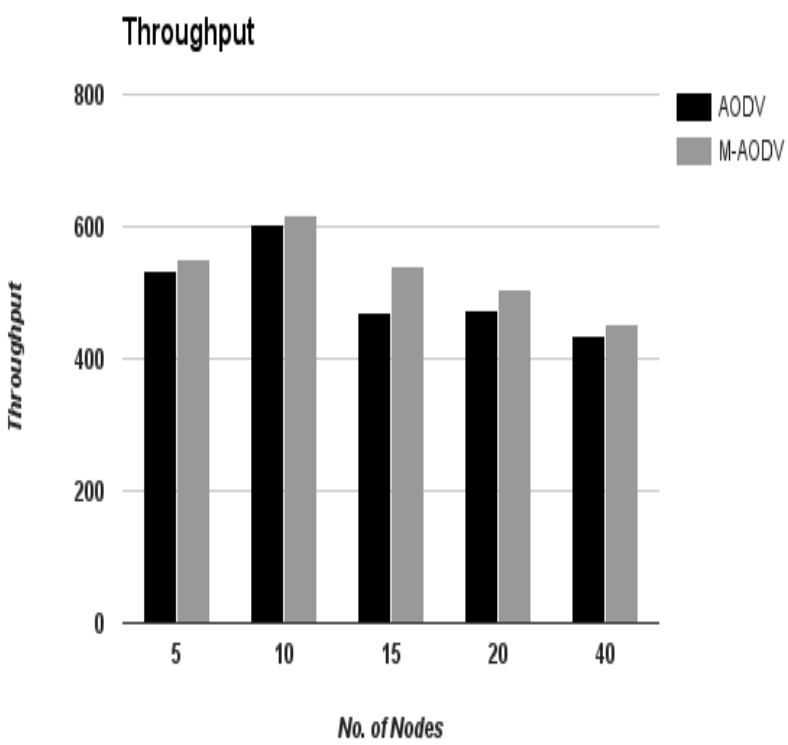

Figure 2: Throughput with respect to number of nodes

2. End to End Delay: The graph represents the End to End delay analysis with respect to number of nodes. The graph represents the decrease in end to end delay in modified AODV when compared with the earlier AODV protocol. The decrease in end to end delay clearly shows that the proposed system reduces the congestion problem and enhances the performance of the network.

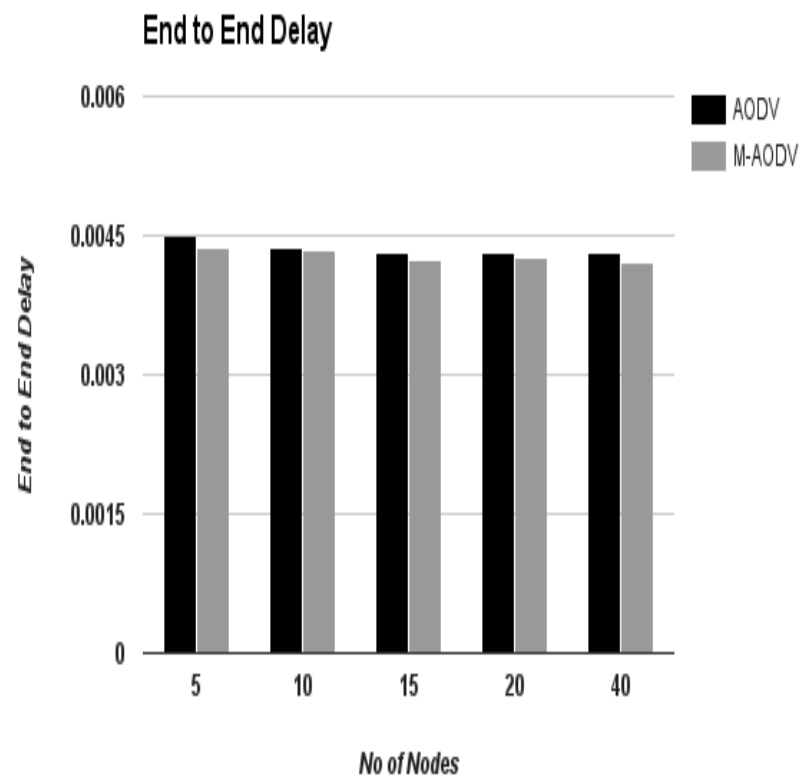

Figure 3: End to End Delay with respect to Number of Nodes

\section{CONCLUSION}

Congestion is the main issue in Wireless Mesh Networks which leads to packet loss and performance degradation of the network. In this work, an algorithm using buffers is implemented in which the number of packets related to each destination present in the buffer is checked against a certain threshold. The proposed work reduces the congestion by adopting the buffer scheme in a modulated form. Once congestion is detected, the congested route is not used for some time as the data is forwarded through alternate routes. The simulation is carried out in NS-2 simulator and different parameters of the network have been considered and a comparative study is done. The simulation results conclude that the proposed algorithm performs much better and improves the network performance by increasing Throughput and decreasing End to End Delay. Controlling Congestion in WMN is an interesting area of research. Though, In this research work, Congestion is reduced by applying technique of buffers in a modulated way, still there is a need to make efforts in this area. Further research is required to completely study about congestion and more efforts are required to control it to increase network performance.

\section{ACKNOWLEDGMENTS}

I would like to thank my guides Prof (Dr.) Paramjeet Singh and Prof (Dr.) Shaveta for their invaluable guidance throughout the course of this Research work. I am very grateful to them for their insightful and detailed comments and suggestions, which have helped me to improve my perceptive to complete my work entitled "Congestion Control in Wireless Mesh Networks". My signs of gratitude go out to my family and my friends, who had encouraged \& inspired me with their blessings and consistent coordination. My acknowledgements would not be complete without expressing my personal belief in and gratitude towards God, our creator. None of this would have been possible without His blessings. 


\section{REFERENCES}

[1] I. F. Akyildiz, "A survey on wireless mesh networks," IEEE Commun. Mag., vol. 43, no. 9, pp. S23-S30, 2005.

[2] G. Maheshwari, M. Gour, and U. K. Chourasia, "A Survey on Congestion Control in MANET," Int. J. Comput. Sci. Inf. Technol., vol. 5, no. 2, pp. 998-1001, 2014.

[3] T. Minhas, "Performance Enhancement of AODV Routing Protocol in Wireless Mesh Networks," Int. J. Inf. Electron. Eng., vol. 4, no. 6, 2014.

[4] B. Bhatia and N. Sood, "AODV based Congestion Control Protocols: Review," vol. 5, no. 3, pp. 45704575, 2014.

[5] B. Ramesh, "CA-AODV: Congestion Adaptive AODV Routing Protocol for Streaming Video in Mobile Ad Hoc Networks," Int'l J. Commun. Netw. Syst. Sci., vol. 01, no. 04, pp. 322-328, 2008.

[6] D. a. Tran and H. R. H. Raghavendra, "Congestion Adaptive Routing in Mobile Ad Hoc Networks," IEEE
Trans. Parallel Distrib. Syst., vol. 17, no. 11, pp. 1294 1305, 2006.

[7] V. K. Sharma and S. S. Bhadauria, "Mobile Agent Based Congestion Control Using AODV Routing Protocol Technique for Mobile Ad-Hoc Network," Int. J. Wirel Mob. Networks(IJWMN), vol. 4, no. 2, pp. 299-314, 2012.

[8] S. Mani and R. Ponraj, "Optimization With Congestion Aware Routing In Mesh Topology," vol. 2, no. 2, pp. 16, 2014.

[9] H. K. Rath, "Cross Layer based Congestion Control in Wireless Networks," vol. 14, no. 2, pp. 65-80, 2014.

[10] C. H. P. Reddy, J. Gopal, and A. K. Sangaiah, "Efficient Bandwidth Utilization with Congestion Control for Wireless Mesh Networks," Int. J. Sci. Technol., vol. 7, no. November, pp. 1780-1787, 2014.

[11] M. Ad, "ECA1RP : An Efflicient Congestion Adative Routing," 6th Int. Conf. ITS Telecommun. Proc., pp. 715-718, 2006 